\title{
Compressed and Stealth SUSY searches with ATLAS and CMS
}

\author{
Giovanni Zevi Della Porta, on behalf of the ATLAS and CMS Collaborations* \\ University of California at San Diego \\ E-mail: gzeviecern.ch
}

\begin{abstract}
Searches for compressed and Stealth supersymmetry models present new and interesting challenges with respect to traditional searches. We discuss recent results of the ATLAS and CMS collaborations based on the first LHC dataset collected at a center-of-mass energy of $13 \mathrm{TeV}$. Several methods are used to select final states sensitive to compressed models, including initial-state radiation jets and soft leptons. No excesses are observed over the standard model background predictions, and the results are used to set limits on compressed models of gluino and squark pair-production.
\end{abstract}

Fourth Annual Large Hadron Collider Physics

13-18 June 2016

Lund, Sweden

${ }^{*}$ Speaker. 


\section{Introduction}

In 2015, at the start of Run 2 of the LHC, proton-proton collisions at a center-of-mass energy of $13 \mathrm{TeV}$ were recorded for the first time by the ATLAS [1] and CMS [2] experiments. The data, corresponding to an integrated luminosity of $3.2 \mathrm{fb}^{-1}$ in ATLAS and $2.3 \mathrm{fb}^{-1}$ in CMS, were analyzed to search for signs of new physics, and they were found to be in good agreement with the standard model (SM). The results were used to set limits on beyond-the-SM (BSM) scenarios, and several final states contributed to an improved sensitivity to models of compressed supersymmetry (SUSY) $[3,4,5,6,7,8,9,10]$. For models of Stealth SUSY, the best available limits at the time of this report are based on the $8 \mathrm{TeV}$ Run 1 of the LHC [11], so they are not discussed here.

For our purposes, compressed SUSY ${ }^{1}$ refers to any simplified SUSY model (SMS) [13] in which the mass difference between the next-to-lightest SUSY particle (NLSP) and the sum of its decay products - the lightest SUSY particle (LSP) and the SM particle involved in the specific decay - is small. The small difference between $m_{\mathrm{NLSP}}$ and the sum of its decay products $\left(m_{\mathrm{SM}}+\right.$ $\left.m_{\text {LSP }}\right)$ results in the daughter particles having very small momenta in the NLSP frame. When the NLSP is boosted, most of its boost is inherited by the more massive of the daughter particles, giving two distinct behaviors for the two asymmetric configurations: models where $m_{\mathrm{NLSP}} \approx m_{\mathrm{LSP}}$ and $m_{\mathrm{SM}} \approx 0$ can give rise to final states with large missing transverse momentum $\left(E_{\mathrm{T}}^{\mathrm{miss}}\right)$ and low momentum SM particles, while the opposite is true for models where $m_{\mathrm{LSP}} \approx 0$.

Stealth SUSY, as proposed here [14], and discussed by M. Reece during this conference, is an extension of SUSY which includes a new sector with at least two particles, the scalar singlet $X$ and its fermion superpartner $\tilde{X}$. SUSY-breaking in the new sector is small, due to the small coupling with the SUSY-breaking fields of the visible sector, leading to $m_{\tilde{X}} \approx m_{X}$. This small mass difference is similar to the one of compressed SUSY, but it is an intrinsic feature of the theory rather than being realized only in a small part of the parameter space. Typically, the $\tilde{X}$ can be produced in an R-parity conserving SUSY decay, such as $\tilde{\chi}_{1}^{ \pm} \rightarrow W^{ \pm} \tilde{X}$, which can be followed by $\tilde{X} \rightarrow X+$ LSP and the $X$ decaying to two SM particles, for example $X \rightarrow g g$. Because of the small mass difference between $\tilde{X}$ and $X$, the $X$ carries most of the boost of the parent $\tilde{X}$, the almost-massless LSP (generally a gravitino, $\tilde{G}$ ) is left with little transverse momentum, and the resulting final state has small $E_{\mathrm{T}}^{\text {miss }}$.

The Run 2 results on compressed SUSY focus on strong production, where the energy increase provides the largest gains with respect to Run 1, so the pair-produced SUSY particles are either gluinos $(\tilde{g})$ or squarks $(\tilde{b}$ and $\tilde{t})$. In the SMS considered, the LSP is always a neutralino $\left(\tilde{\chi}_{1}^{0}\right)$, and there can be an intermediate particle, $\tilde{\chi}_{1}^{ \pm}$or $\tilde{t}$, with mass between the produced particle and the LSP. The final states considered, all with large $E_{\mathrm{T}}^{\text {miss }}$ except for the last one, are: the 'monojet' final state, characterized by a high $p_{\mathrm{T}}$ jet recoiling against $E_{\mathrm{T}}^{\text {miss }}$; the multijet with b-tags final state; the 1-soft-lepton final state, with lepton $p_{\mathrm{T}}$ below 20 or $35 \mathrm{GeV}$; and the same-sign dilepton final state, including lepton $p_{\mathrm{T}}$ below $25 \mathrm{GeV}$. For each final state, we briefly describe the event selection and we present a subset of the results plots. Among these analyses, we include more details for the 1-soft-lepton analysis in CMS, as it was shown for the first time at LHCP16.

\footnotetext{
${ }^{1}$ Compressed SUSY, as originally proposed in [12], is characterized by a small ratio of the squark and gluino masses to the lightest supersymmetric particle (LSP) mass.
} 


\section{Searches in the monojet final state}

The ATLAS and CMS monojet searches are reported in [3] and [4]. We discuss the ATLAS search, as it includes interpretations in compressed SUSY models. The monojet final state is characterized by a high- $p_{\mathrm{T}}$ initial-state radiation (ISR) jet recoiling against a system whose mass is dominated by invisible particles. The invisible particles then receive most of the boost, causing a large $E_{\mathrm{T}}^{\text {miss }}$ signature in the direction opposite to the ISR jet. This analysis focuses on the tail of the $E_{\mathrm{T}}^{\text {miss }}$ distribution in events with a high- $p_{\mathrm{T}}$ central jet $\left(p_{\mathrm{T}}>250 \mathrm{GeV}, \eta<2.4\right.$ ). To reduce SM backgrounds from electroweak and multijet processes, events with leptons are vetoed, as are events with a jet aligned with the $E_{\mathrm{T}}^{\text {miss }}$ direction $\left(\Delta \phi\left(\right.\right.$ jet,$\left.\left.E_{\mathrm{T}}^{\text {miss }}\right)>0.4\right)$ ). Events with up to four jets with $p_{\mathrm{T}}>30 \mathrm{GeV}$ are considered. Signal regions are defined as a function of $E_{\mathrm{T}}^{\text {miss }}$, with the tightest signal region $\left(E_{\mathrm{T}}^{\text {miss }}>700 \mathrm{GeV}\right)$ containing 185 observed events and $167 \pm 20$ events predicted from SM backgrounds.

The results are shown as a function of $E_{\mathrm{T}}^{\text {miss }}$ in Figure 1 (left), where the agreement between data and SM prediction is observed. An interpretation in terms of compressed SUSY is shown in Figure 1 (right). The interpretation is based on a model of top squark pair-production $(p p \rightarrow \tilde{t} \tilde{t})$, followed by a flavor-changing decay of the top squark, $\tilde{t} \rightarrow c \tilde{\chi}_{1}^{0}$. Limits (at the $95 \%$ confidence level) on the number of signal events are first converted to cross-section limits based on the efficiency and acceptance predicted by the SUSY model, then to mass limits based on the predicted SUSY cross-section. The limits are shown in the $m_{\tilde{t}}-m_{\tilde{\chi}_{1}^{0}}$ plane, reaching their highest value along the diagonal where $m_{\tilde{t}}-m_{\tilde{\chi}_{1}^{0}}=5 \mathrm{GeV}$, for masses around $320 \mathrm{GeV}$. The limits become weaker away from the diagonal, as the charm quark inherits more of the $\tilde{t}$ boost. An identical limit is produced for the model of bottom squark production, $p p \rightarrow \tilde{b} \tilde{b}$ followed by $\tilde{b} \rightarrow b \tilde{\chi}_{1}^{0}$, while the limits for light squark production ( $p p \rightarrow \tilde{q} \tilde{q}$ ) can reach higher mass values (up to $608 \mathrm{GeV}$ ), depending on the mass degeneracy of the light squarks.
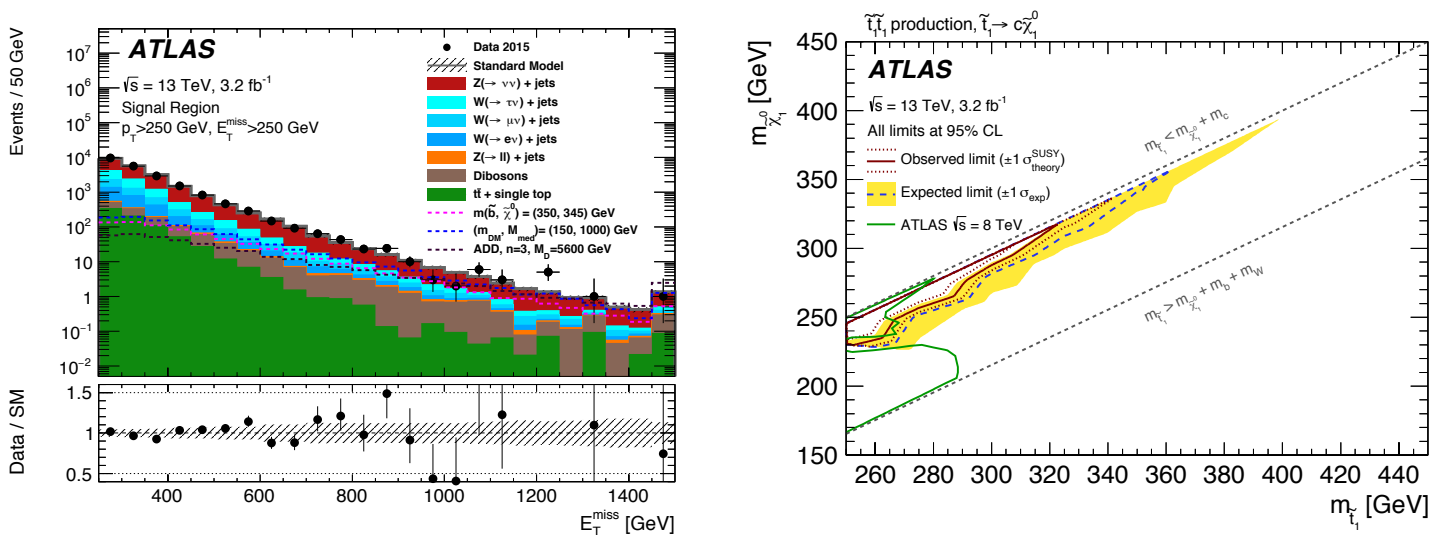

Figure 1: (right) Comparison of estimated background and observed data yields as a function of $E_{\mathrm{T}}^{\mathrm{miss}}$ for the monojet final state in ATLAS [3]. The corresponding distribution for a compressed SUSY signal is shown as a dashed blue line. (left) Interpretation of the result in terms of exclusion limits for top squark production in the $m_{\tilde{t}}-m_{\tilde{\chi}_{1}^{0}}$ plane. The excluded area is enclosed by the solid red contour. 


\section{Searches in the multi-jet with b-tags final state}

The ATLAS and CMS searches in the mutlijet with b-tags final state are reported in [5] and [6]. The searches target bottom squark production, $p p \rightarrow \tilde{b} \tilde{b}$ followed by $\tilde{b} \rightarrow b \tilde{\chi}_{1}^{0}$, and they rely on a similar event selection, with dedicated search regions for compressed models. Events in the compressed signal regions are required to have a non-b-tagged central jet, with $p_{\mathrm{T}}>250(300) \mathrm{GeV}$ in CMS (ATLAS), a second jet with $p_{\mathrm{T}}>60(50) \mathrm{GeV}$, and up to one additional jet. Events with a leptons are vetoed, as are event with a jet aligned with the $E_{\mathrm{T}}^{\text {miss }}$ direction. Additionally, backgrounds are reduced in the ATLAS search using a harsh $E_{\mathrm{T}}^{\text {miss }}$ requirement $(400 \mathrm{GeV})$ and requiring a significant ratio of $E_{\mathrm{T}}^{\text {miss }}$ to the scalar sum of the $E_{\mathrm{T}}^{\mathrm{miss}}$ and the $p_{\mathrm{T}}$ of jets $\left(E_{\mathrm{T}}^{\mathrm{miss}} / m_{\text {eff }}>\right.$ 0.25). In the CMS search, the $E_{\mathrm{T}}^{\text {miss }}$ requirement is lower $(200 \mathrm{GeV})$, but the transverse mass formed by $E_{\mathrm{T}}^{\text {miss }}$ and each of the two leading jets is required to be larger than $200 \mathrm{GeV}$. Both ATLAS and CMS include a signal region with two b-tagged jets, and CMS additionally includes signal regions with one or zero b-tagged jets, and higher $E_{\mathrm{T}}^{\text {miss }}$ requirements.

The data yields agree well with SM predictions for both the ATLAS and CMS analyses, and the results are interpreted in terms of bottom squark production in the $m_{\tilde{b}}-m_{\tilde{\chi}_{1}^{0}}$ plane. The CMS analysis also includes an interpretation for top squark production followed by the decay $\tilde{t} \rightarrow c \tilde{\chi}_{1}^{0}$, taking advantage of the non b-tagged signal regions. The compressed signal regions described above play a significant role in the part of the plane closer to the diagonal, while the non-compressed signal regions cover the bulk of the plane where $m_{\tilde{b}} \gg m_{\tilde{\chi}_{1}^{0}}$. The limits are shown in Figure 2 for ATLAS (left) and CMS (right). The two analyses reach similar masses across the plane, with mass limits slowly weakening for $\Delta m<300 \mathrm{GeV}$, and neither is as effective as the monojet analysis in probing the extremely compressed region $(\Delta m \approx 10 \mathrm{GeV})$.
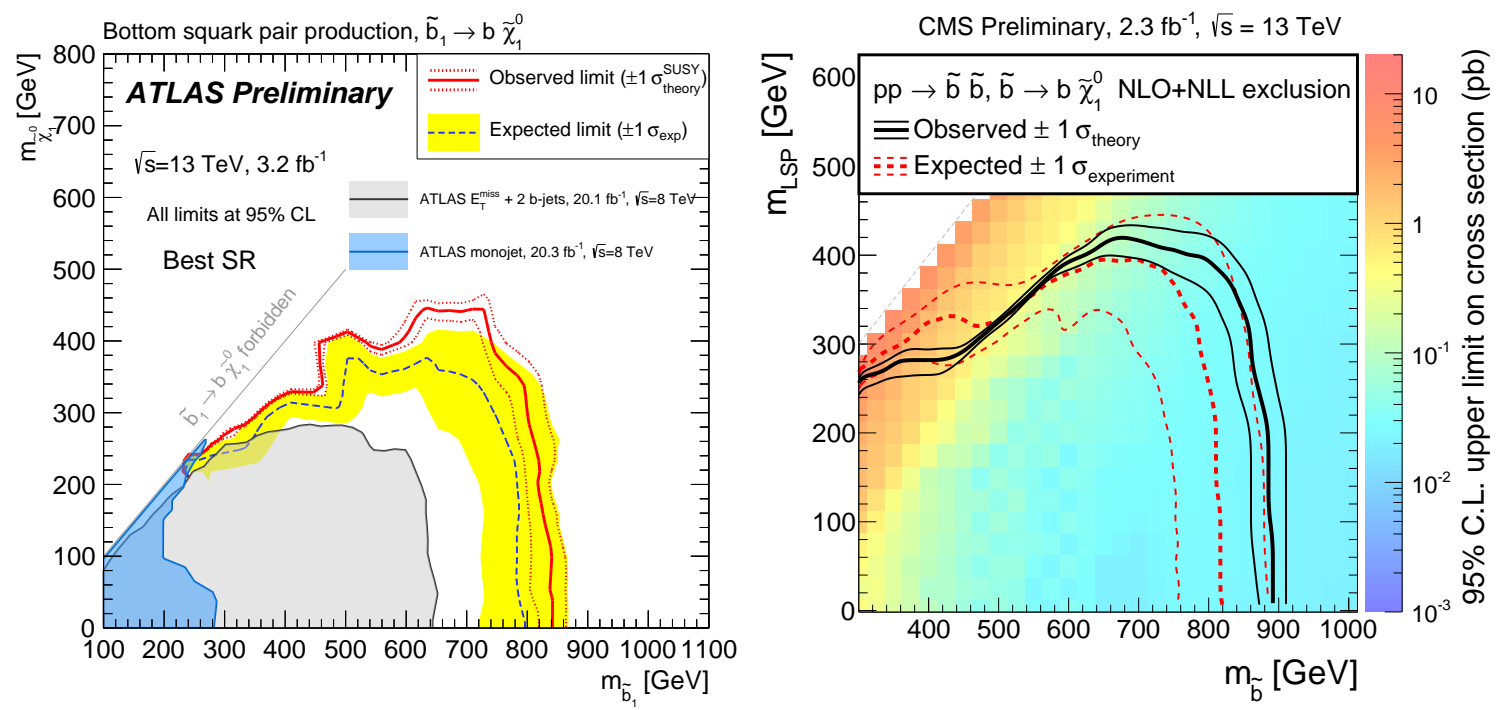

Figure 2: Interpretation of the results of the multijet search with b-tags in terms of exclusion limits for bottom squark production in the $m_{\tilde{b}}-m_{\tilde{\chi}_{1}^{0}}$ plane. The ATLAS limit [5] is shown on the left, while the CMS limit [6], where the color code represent the excluded cross-section for this model, is shown on the right. 


\section{Searches in the 1-soft-lepton final state}

The ATLAS and CMS searches in the 1-soft-lepton final state are reported in [7] and [8]. This final state is sensitive to models with off-shell $W$ bosons, typically generated when the top squark or chargino mass is very close to the mass of the neutralino LSP. The off-shell $W$ can produce a soft lepton, while the more massive LSP inherits most of the boost causing a $E_{\mathrm{T}}^{\text {miss }}$ signature. In terms of SM backgrounds, requiring one lepton rejects the processes which include a $Z \rightarrow v v$ decayan irreducible background in zero-lepton analyses-while requiring that the lepton be soft rejects the bulk of all processes with a leptonic decay of the $W$ boson.

The ATLAS search considers leptons with a $p_{\mathrm{T}}$ of 6-35 GeV, but it also includes signal regions with a lepton of $p_{\mathrm{T}}>35 \mathrm{GeV}$ to also cover the non-compressed models. The CMS search uses leptons with a $p_{\mathrm{T}}$ of 5-20 GeV, and it is designed to be complementary to other searches which use high- $p_{\mathrm{T}}$ leptons [15]. Events are categorized in signal regions based on jet-related quantities such as the number of jets, their individual $p_{\mathrm{T}}$, their scalar sum $H_{\mathrm{T}}$, and the $E_{\mathrm{T}}^{\text {miss }}$. In addition, the ATLAS search uses the jet aplanarity variable to remove relatively planar background-like events, and the CMS search uses the number and $p_{\mathrm{T}}$ of b-tagged jets to categorize events. Finally, the transverse mass between the lepton and the $E_{\mathrm{T}}^{\text {miss }}, m_{\mathrm{T}}\left(p_{\mathrm{T}}^{\ell}, E_{\mathrm{T}}^{\text {miss }}\right)$, is used by both analyses to further reject SM backgrounds with one leptonic $W$. Overall, the ATLAS analysis focuses on two signal regions, one with large $E_{\mathrm{T}}^{\text {miss }}$ and $m_{\mathrm{T}}\left(E_{\mathrm{T}}^{\text {miss }}>530 \mathrm{GeV}, m_{\mathrm{T}}>100 \mathrm{GeV}\right)$, and one with a large number of jets and $H_{\mathrm{T}}\left(n_{\text {jet }} \geq 5, H_{\mathrm{T}}>1100 \mathrm{GeV}\right)$, while the CMS analysis searches for excesses in 63 signal regions covering the entire available phase space after a $E_{\mathrm{T}}^{\text {miss }}$ cut motivated by the trigger selection $\left(E_{\mathrm{T}}^{\text {miss }}>200 \mathrm{GeV}\right)$.

As shown in Figure 3 for ATLAS (top) and CMS (bottom), the data yields agree well with the SM background predictions in both analyses and no significant excess is observed. Both results are interpreted in terms of limits on gluino pair-production decaying through an intermediate chargino: $\tilde{g} \rightarrow q q \tilde{\chi}_{1}^{ \pm}$followed by $\tilde{\chi}_{1}^{ \pm} \rightarrow W \tilde{\chi}_{1}^{0}$, as shown in Figure 4 (top and bottom left). In the ATLAS interpretation, the chargino mass is chosen to be the average of the gluino and neutralino masses, $m_{\tilde{\chi}_{1}^{ \pm}}=\left(m_{\tilde{g}}+m_{\tilde{\chi}_{1}^{0}}\right) / 2$. As a consequence, the compressed spectrum is only found close to the diagonal, and the limit plot shows clearly the separate reach of the compressed and non-compressed regions. In the CMS interpretation, the chargino mass is chosen to be $20 \mathrm{GeV}$ higher than the neutralino mass $m_{\tilde{\chi}_{1}^{ \pm}}=m_{\tilde{\chi}_{1}^{0}}+20 \mathrm{GeV}$. Due to this choice, the $W$ boson is off-shell throughout the $m_{\tilde{g}}-m_{\tilde{\chi}_{1}^{0}}$ plane, and the soft lepton analysis plays a bigger role as long as $m_{\tilde{\chi}_{1}^{0}}$ is large. For smaller neutralino masses, the $W$ inherits more of the chargino boost in the decay $\tilde{\chi}_{1}^{ \pm} \rightarrow W \tilde{\chi}_{1}^{0}$, resulting in higher $p_{\mathrm{T}}$ leptons that fail the signal region selection.

In addition to the $\tilde{g} \rightarrow q q \tilde{\chi}_{1}^{ \pm}$model, the CMS analysis also provides an interpretation for top squark pair production in the region of $\left(m_{\tilde{t}}-m_{\tilde{\chi}_{1}^{0}}\right)<m_{W}$. In this model, the top squark decays in a flavor-conserving four-body final state, $\tilde{t} \rightarrow b f f^{\prime} \tilde{\chi}_{1}^{0}$, with $f f^{\prime}$ representing $\ell v$ or $q q^{\prime}$ according to $W$-like branching fractions. The limit, shown in Figure 4 (bottom), is the first Run 2 result for this model, and reaches further than any Run 1 result despite the luminosity being lower by an order of magnitude. 

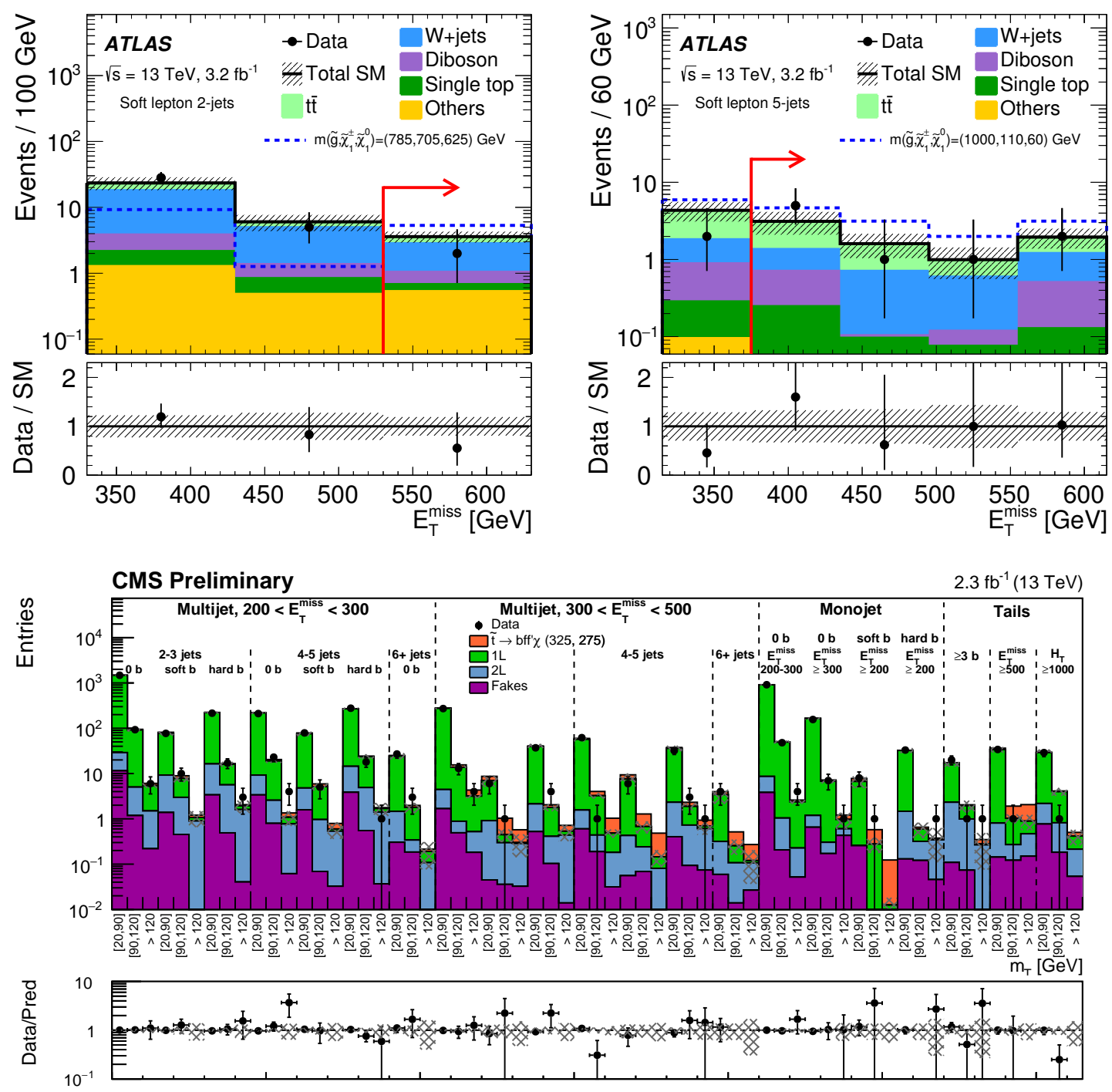

Figure 3: (top) Comparison of estimated background and observed data yields in the ATLAS 1-soft-lepton signal regions [7], marked by red arrows in the $E_{\mathrm{T}}^{\text {miss }}$ distribution. The corresponding distribution for two SUSY signals are shown as dashed blue lines. (left) Comparison of estimated background and observed data yields in the signal regions of the CMS 1-soft-lepton analysis [8]. The expected yields for a SUSY signal are shown as an orange stacked histogram.

\section{Searches in the same-sign dilepton final state}

The ATLAS and CMS searches in the same-sign dilepton final state are reported in [9] and [10]. This very clean final state is populated only by rare SM processes, such as $W Z$ and $t t W$ production, as well as by reducible backgrounds such as non-prompt or misreconstructed leptons. The SUSY interpretations generally assume pair-produced gluinos, with at least one $W$ boson in each decay chain: since gluinos are neutral, the leptons of the two decay chains can have the same charge, or multiple leptons can be produced from each gluino, creating same-sign dilepton pairs. As in the 

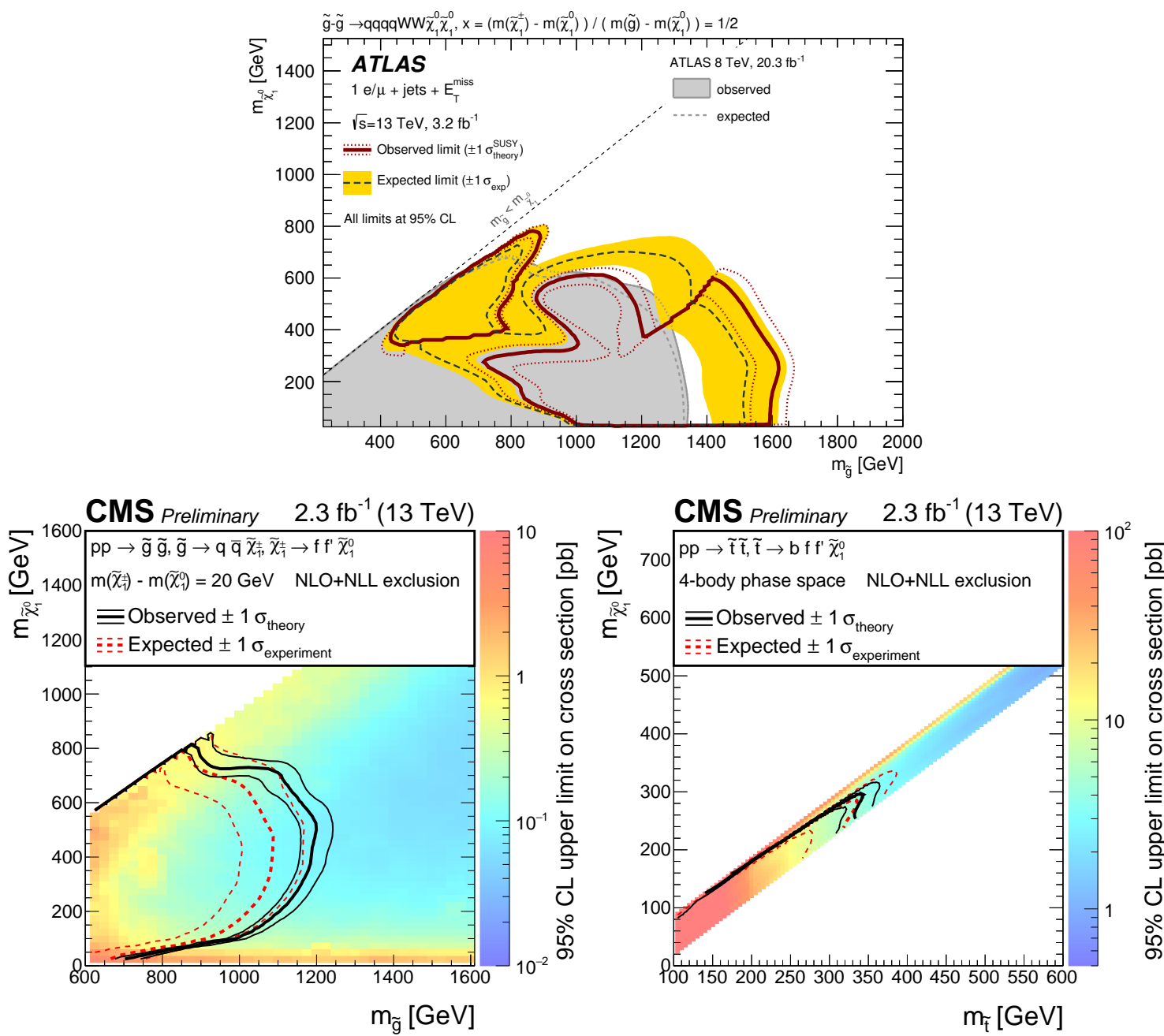

Figure 4: (top) Interpretation of the results of the ATLAS 1-lepton analysis [7] in terms of exclusion limits for gluino production in the $m_{\tilde{g}}-m_{\tilde{\chi}_{1}^{0}}$ plane, for $m_{\tilde{\chi}_{1}^{ \pm}}=\left(m_{\tilde{g}}+m_{\tilde{\chi}_{1}^{0}}\right) / 2$. The triangular area closer to the diagonal line shows the signal points excluded by the soft lepton signal regions. (bottom left) Interpretation of the results of the CMS 1-soft-lepton analysis [8] in terms of exclusion limits for gluino production in the $m_{\tilde{g}}-m_{\tilde{\chi}_{1}^{0}}$ plane, for a different assumption of the chargino mass: $m_{\tilde{\chi}_{1}^{ \pm}}=m_{\tilde{\chi}_{1}^{0}}+20 \mathrm{GeV}$. (bottom right) Interpretation of the CMS result in terms of exclusion limits for top squark production in the $m_{\tilde{t}}-m_{\tilde{\chi}_{1}^{0}}$ plane.

1-soft-lepton analysis, soft leptons can be produced if the $W$ bosons are off-shell.

We discuss the CMS search, as it includes signal regions sensitive to compressed SUSY models. Specifically, in addition to signal regions with two high- $p_{\mathrm{T}}$ leptons, the CMS search includes regions where one or both leptons are in the range $10(15)<p_{\mathrm{T}}<25 \mathrm{GeV}$ for muons (electrons). The analysis uses 66 signal region to cover the entire available phase space of events with same-sign dileptons, at least two jets, $E_{\mathrm{T}}^{\text {miss }}>50 \mathrm{GeV}$. When both leptons have $p_{\mathrm{T}}<25 \mathrm{GeV}$, an $H_{\mathrm{T}}>300 \mathrm{GeV}$ requirement is also applied, motivated by the trigger. Events are classified based on properties such as the number of jets and b-jets, the $E_{\mathrm{T}}^{\text {miss }}$ and $H_{\mathrm{T}}$, and the minimum transverse mass: $m_{\mathrm{T}}^{\min }=\min \left[m_{\mathrm{T}}\left(p_{\mathrm{T}}^{\ell 1}, E_{\mathrm{T}}^{\mathrm{miss}}\right), m_{\mathrm{T}}\left(p_{\mathrm{T}}^{\ell 2}, E_{\mathrm{T}}^{\text {miss }}\right)\right]$. 
The data yields agree well with SM background predictions across all signal regions, and limits are set on the masses and cross-sections for several SUSY models. The role of the compressed signal regions can be seen, for example, when comparing models of gluino pair-production with a different number of soft and high- $p_{\mathrm{T}}$ leptons. In Figure 5, three limit plots of gluino pairproduction are compared: one (left) where the gluino decay follows $\tilde{g} \rightarrow t \bar{t} \tilde{\chi}_{1}^{0}$, with potentially two high- $p_{\mathrm{T}}$ leptons for each gluino; one (middle) where an intermediate top squark with a mass only $20 \mathrm{GeV}$ above the neutralino $\left(m_{\tilde{t}}-m_{\tilde{\chi}_{1}^{0}}=20 \mathrm{GeV}\right)$ yields potentially one high- $p_{\mathrm{T}}$ and one soft lepton for each gluino through the decays $\tilde{g} \rightarrow t \tilde{t}$ and $\tilde{t} \rightarrow b f f^{\prime} \tilde{\chi}_{1}^{0}$; and one (right) where the decay only includes one top quark, hence one potential lepton, for each gluino. Comparing the left and right exclusion curves, it is clear that losing two of the potential high- $p_{\mathrm{T}}$ leptons corresponds to a $200 \mathrm{GeV}$ lower reach in terms of gluino mass. The middle plot, however, shows that recovering the two soft leptons in models that include them can reduce this loss by a factor of two.
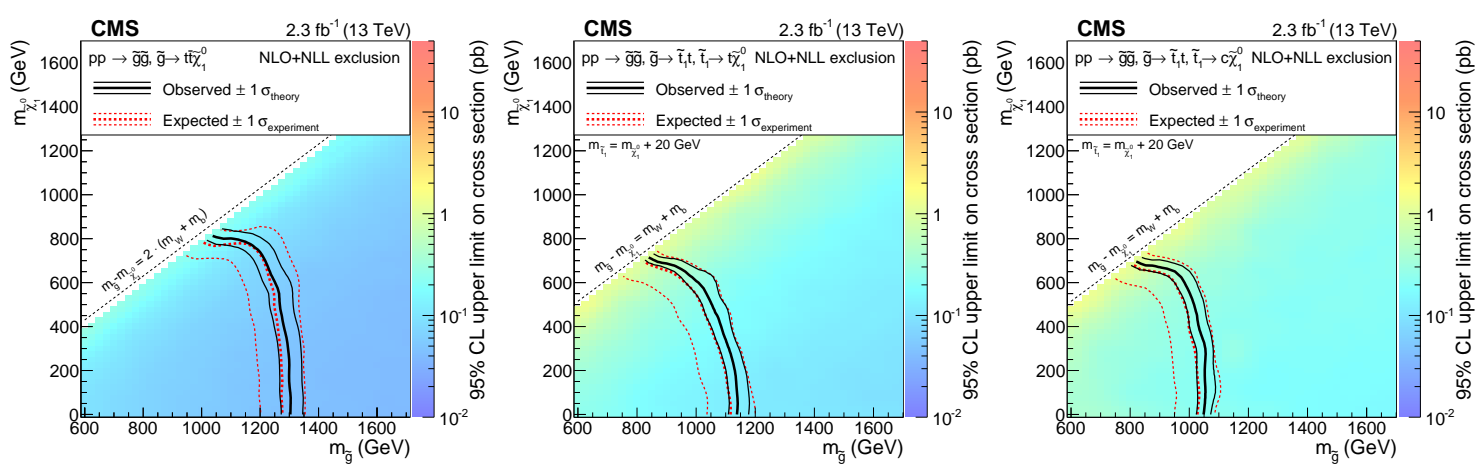

Figure 5: Interpretations of the CMS same-sign dilepton results [10] in terms of exclusion limits for several models of gluino pair-production in the $m_{\tilde{g}}-m_{\tilde{\chi}_{1}^{0}}$ plane. In the left plot, each gluino decays directly to a pair of top quarks: $\tilde{g} \rightarrow t \bar{\tau} \tilde{\chi}_{1}^{0}$. In the middle and right plots, the decay proceeds through a top squark, with mass only slightly higher than the LSP $\left(m_{\tilde{t}}-m_{\tilde{\chi}_{1}^{0}}=20 \mathrm{GeV}\right)$. Depending on the model, the top squark can either decay to a four-body final state $b f f^{\prime} \tilde{\chi}_{1}^{0}$ resulting from a very off-shel top quark (middle) or through the flavor-changing decay $\tilde{t} \rightarrow c \tilde{\chi}_{1}^{0}$ (right).

\section{Conclusions}

Several complementary approaches can be used to probe the compressed regions of the SUSY phase space. Recent results by both the ATLAS and CMS collaborations use the 2015 dataset, corresponding to an integrated luminosity of $2.3-3.2 \mathrm{fb}^{-1}$ of $13 \mathrm{TeV}$ proton-proton collisions at the LHC, to search for signs of new physics in final states with an initial-state radiation jet, with or without additional b-jets, as well as final states with one soft lepton or with two same-sign leptons. No excess is observed over the standard model background, and the results are used to set limits on a variety of compressed SUSY models featuring gluino and squark pair-production with small mass differences between the LSP and the next-to-lightest SUSY particle. 


\section{References}

[1] ATLAS Collaboration, JINST 3 S08003 (2008).

[2] CMS Collaboration, JINST 3 S08004 (2008).

[3] ATLAS Collaboration, Phys. Rev. D 94, no. 3, 032005 (2016), arXiv:1604.07773 [hep-ex].

[4] CMS Collaboration, CMS-PAS-EXO-16-013, https://http://cds.cern.ch/record/2148032.

[5] ATLAS Collaboration, ATLAS-CONF-2015-066, https://http://cds.cern.ch/record/2114833.

[6] CMS Collaboration, CMS-PAS-SUS-16-001, https://cds.cern.ch/record/2140975.

[7] ATLAS Collaboration, arXiv:1605.04285 [hep-ex].

[8] CMS Collaboration, CMS-PAS-SUS-16-011, https://cds.cern.ch/record/2161097.

[9] ATLAS Collaboration, Eur. Phys. J. C 76, no. 5, 259 (2016), arXiv:1602.09058 [hep-ex].

[10] CMS Collaboration, Eur. Phys. J. C 76, no. 8, 439 (2016), arXiv:1605.03171 [hep-ex].

[11] CMS Collaboration, Phys. Lett. B 743, 503 (2015), [arXiv:1411.7255 [hep-ex]].

[12] S. P. Martin, Phys. Rev. D 75, 115005 (2007), hep-ph/0703097 [HEP-PH].

[13] LHC New Physics Working Group, J. Phys. G 39, 105005 (2012), arXiv:1105.2838 [hep-ph].

[14] J. Fan, M. Reece and J. T. Ruderman, JHEP 1111, 012 (2011), [arXiv:1105.5135 [hep-ph]].

[15] CMS Collaboration, CMS-PAS-SUS-15-006, https://cds.cern.ch/record/2140638. 